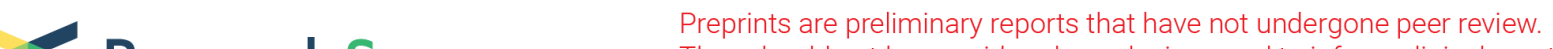 Research Square They should not be considered conclusive, used to inform clinical practice, or referenced by the media as validated information.
}

\section{miR-211-3p enhances induction chemotherapy insensitivity by upregulating CSF2/CCL20/TNF signaling in hypopharyngeal squamous cell carcinoma}

\section{Lingwa Wang}

Beijing Tongren Hospital Department of Otorhinolaryngology and Head and Neck Surgery: Beijing Tongren Hospital

\section{Ru Wang}

Beijing Tongren Hospital Department of Otorhinolaryngology and Head and Neck Surgery: Beijing Tongren Hospital

\section{Tianqiao Huang}

Beijing Tongren Hospital Department of Otorhinolaryngology and Head and Neck Surgery: Beijing Tongren Hospital

\section{Yifan Yang}

Beijing Tongren Hospital Department of Otorhinolaryngology and Head and Neck Surgery: Beijing Tongren Hospital

\section{Ling Feng}

Beijing Tongren Hospital Department of Otorhinolaryngology and Head and Neck Surgery: Beijing Tongren Hospital

Jugao Fang ( $\square$ fangjugao2@ccmu.edu.cn )

Beijing Tongren Hospital https://orcid.org/0000-0002-0188-7120

\section{Research Article}

Keywords: hypopharyngeal squamous cell carcinoma (HSCC), induction chemotherapy (IC), miR-211-3p

Posted Date: February 16th, 2022

DOI: https://doi.org/10.21203/rs.3.rs-1322423/v1

License: (a) (1) This work is licensed under a Creative Commons Attribution 4.0 International License. Read Full License 


\section{Abstract}

Purpose To investigate the potential mechanisms of miR-211-3p on induction chemotherapy (IC) sensitivity in hypopharyngeal squamous cell carcinoma (HSCC).

Methods qRT-PCR was assessed to compare the miR-211-3p expression between IC sensitive and insensitive tumor tissues. The MTT assay was performed to analyze cell proliferation and viability to paclitaxel after alteration of miR-211-3p. Flow cytometry assay was conducted to explore cell apoptosis. Transwell assay was used to explore the effect of miR-211-3p on cell migration. Transcriptome sequencing was then performed to select differentially expressed genes (DEGs) after over-expression of miR-211-3p. GO and KEGG enrichment analyses were conducted to annotate DEGs. PPI analysis was conducted to screen candidate genes. The differential expression and survival status of candidate genes were further validated in TCGA-HNSCC data. The single sample GSEA method was used to investigate the association between downstream genes and immune cell infiltration.

Results MiR-211-3p was up-regulated in IC insensitive larynx-hypopharyngeal tumor tissues. Overexpression of miR-211-3p promoted cell proliferation and migration, and inhibited apoptosis.

The IC50 value of miR-211-3p overexpression (OE) group was significantly higher than negative control (NC) group treated with paclitaxel, suggesting miR-211-3p enhanced IC insensitivity in HSCC. We found 778 DEG after over-expression of miR-211-3p and 11 significant genes were then identified. Finally, CSF2 and CCL20 were validated to be significantly high expressed and associated with poorer overall survival in head and neck squamous cell carcinoma, which were involved in TNF signaling pathway and then regulated immune cell infiltration.

Conclusion The miR-211-3p could promote HSCC progression and upregulates CSF2/CCL20/TNF signaling to promote IC insensitivity in HSCC, which may provide new ideas for HSCC therapy.

\section{Introduction}

Head and neck cancer is the sixth most common malignant tumor worldwide in 2018, with 890,000 new cases and 450,000 death cases [1]. Hypopharyngeal squamous cell carcinoma (HSCC) is one of the classical head and neck squamous cell carcinoma (HNSCC), which is highly aggressive and invasive, prone to local recurrence and metastasis. The 5-year overall survival rate of HSCC patients is approximately $40 \%$ [2]. About $60 \%$ of patients presented with advanced (stage III or IV) disease at clinical diagnosis [3], which would greatly affect patients' quality of life after the treatment. In recent years, induction chemotherapy (IC) followed by surgery and/or concurrent chemoradiotherapy (CCR) is a strong option for inoperable diseases. The application of IC can effectively decrease the tumor volume, increase larynx preservation rates and overall survival rates, yet, some hypopharyngeal cancer patients exhibited no response to IC [4]. 
MicroRNAs (miRNAs) are small endogenous RNAs (19-25 nucleotides long) that regulate gene-expression post-transcriptionally [5]. The abnormal expression of some miRNAs can alter tumor cells chemosensitivity and reverse multi-drug resistance. For instance, the miR-155/TP53 negative feedback loop is involved in resistance to multiple chemotherapeutic drugs used in lung cancer [6]. MiR-10bmediated PPARY inhibition enhances cisplatin resistance by activating AKT/mTOR/P70S6K signaling in esophageal cancer [7]. MiR-621 enhances the sensitivity to paclitaxel plus carboplatin chemotherapy by suppressing FBX011 and enhancing P53 activity in breast cancer [8]. MiR-211 is localized on intron 6 of the Trpm1 gene at 15q13-q14, a locus that is frequently lost in cancers. In the previous high throughput sequencing analysis, we found miR-211-3p was high expressed in IC insensitive hypopharyngeal tumor tissues. Nevertheless, the underlying molecular mechanisms of miR-211-3p involved in IC remain poorly understood.

In our study, we demonstrated that miR-211-3p was up-regulated in insensitive larynx-hypopharyngeal tumor tissues to IC by qRT-PCR. In HSCC tumor cells the over-expression of miR-211-3p promoted cell proliferation and migration, and inhibited apoptosis. The IC50 value of miR-211-3p OE group was significantly higher than miR-211-3p NC group treated with paclitaxel. Furthermore, we identified that the high expression of miR-211-3p lead to high CSF2 and CCL20 expression which correlated with poor prognosis in HNSCC.

\section{Methods And Materials}

\section{qRT-PCR}

The relative expression of miR-211-3p in 20 IC sensitive larynx-hypopharyngeal tumor tissues and 13 IC insensitive tissues was assessed by qRT-PCR. Total RNA was extracted from tissues using Trizol reagent (Invitrogen) and then reverse-transcribed to cDNA using miR-211-3p specific primers. Quantitative PCR analysis and data collection were carried out in triplicate for each sample and performed on the $A B I 7500$ Real-Time PCR System (Applied Biosystems, Carlsbad, CA, USA) using the primer pairs (miR-211-3p-F: TTGTACGCAGGGACAGCAAA, miR-211-3p-R: TATGGTTGTTCACGACTCCTTCAC). Relative quantification was achieved according to the $2^{-\triangle \triangle C t}$ relative quantification method.

\section{Cell culture and transfection}

Fadu hypopharyngeal carcinoma cell line weas obtained from Beijing Institute of Otorhinolaryngology. The cells were maintained in DMEM (Gibco) with $10 \% \mathrm{FBS}$ (Hyclone) at a $37^{\circ} \mathrm{C}$ humidified atmosphere containing $5 \% \mathrm{CO} 2$ for a maximum of 8 weeks. miR-211-3p overexpression (OE) and negative control (NC) lentivirus vectors, harboring green fluorescent protein (GFP), were constructed by GeneChem (Shanghai, China). After 16 hours post-transfection, cell culture media was replaced. After 72 hours, the mean percentage of Fadu cells expressing GFP was calculated under a fluorescence microscope (IX70, Olympus, Japan), and successful overexpression was confirmed by qRT-PCR.

\section{MTT cell proliferation assay}


For the MTT assay, cell proliferation was evaluated by adding $20 \mu \mathrm{L} 5 \mathrm{mg} / \mathrm{mL}$ MTT to the culture medium for 5 consecutive days. The absorbance was determined at $490 / 570 \mathrm{~nm}$ by a microplate reader (Tecan infinite, Tecan Group Ltd, Switzerland). The same method was used to detect the tumor cell viability after paclitaxel treatment.

\section{Cell apoptosis assay}

The apoptotic cells were detected using Annexin V-allophycocyanin (Annexin V-APC) apoptosis detection kit (eBioscience, San Diego, CA, USA). The process of culturing infected cells in growth medium is the same as before. After trypsinization and PBS washes, the cells were washed with $1 \times$ binding buffer and centrifuged at $1300 \mathrm{rpm}$ for $3 \mathrm{~min}$. Then, the cell pellet was resuspended in $200 \mu \mathrm{L}$ of $1 \times$ binding buffer, and Fadu cells were stained with $10 \mu \mathrm{L}$ Annexin V-APC in the dark for 15 min and detected by flow cytometry using the FACS system.

\section{Transwell migration assay}

The migration potential of Fadu cells was assessed in 24-well Transwell plates. Briefly, cells $\left(1 \times 10^{5}\right.$, $2 \times 10^{5}, 4 \times 10^{5}, 8 \times 10^{5}$ cells) were suspended in $200 \mu \mathrm{l}$ serum-free medium and added into the upper chamber, $500 \mu \mathrm{L}$ medium was added to the lower chamber. After 20 hours culture, cells on the upper surface were removed and migrant cells on the lower surface were fixed with $500 \mu \mathrm{L}$ methanol for $10 \mathrm{~min}$, and stained with $500 \mu \mathrm{L}$ crystal violet for $20 \mathrm{~min}$. And then the migrated cells were visualized using a microscope (400x magnification).

\section{Transcriptome sequencing and differential expression analysis}

Total RNA was extracted using Trizol reagent according to manufacturer's recommendations, and then RNA quantification and qualification analyses were performed. Transcriptome sequencing was performed on an Illumina Novaseq platform. Differential expression analysis of two groups (over-expression of miR211-3p vs control) was performed using the DESeq2 R package [9]. Significant differentially expressed genes (DEGs) were selected for further analyses with setting the $|\log 2(\mathrm{FC})|>1$ \& P.adj $<0.05$ (FC, fold change).

\section{GO and KEGG enrichment analyses of differentially expressed genes}

Gene Ontology (GO) and KEGG ((Kyoto Encyclopedia of Genes and Genomes) functional enrichment analyses were performed based on the hypergeometric test. The clusterProfiler $\mathrm{R}$ package was used to test the statistical enrichment of DEGs in GO terms and KEGG pathways $[10,11]$. P.adj $<0.05 \&$ qvalue $<$ 0.2 was considered significance.

\section{PPI analysis of differentially expressed genes}


DEGs were uploaded into the STRING online database to further screen significant gene module [12]. Then PPIs with a combined score $>0.7$ (high confidence) were chosen. And then, PPI networks were constructed by the Cytoscape software [13]. The plug-in Molecular Complex Detection (MCODE) was applied to pick out the significant gene modules.

\section{Validation downstream genes in TCGA-HNSCC data}

These candidate genes were further validated at gene expression level and OS (overall survival) time based on TCGA-HNSCC data. Gene expression validation involved in noncorresponding normal samples (502 tumor samples; 44 normal samples) and corresponding normal controls (43 tumor samples and matched normal samples). For the survival analysis, all patients with available OS time data were divided into low and high expression groups by the median gene expression level. $\mathrm{P}<0.05$ is regarded as statistically significant.

\section{Association of downstream genes and immune cell infiltration in HNSCC}

The single-sample gene set enrichment analysis (sSGSEA) method from GSVA R package was used to investigate the association between downstream genes and immune cell infiltration [14, 15]. A total of 24 immune cell types were available to analysis. These immune cells included activated DC (aDC), B cells, CD8 T cells, Cytotoxic cells, DC, Eosinophils, immature DC (iDC) Macrophages, Mast cells, Neutrophils, NK CD56bright cells, NK CD56dim cells, NK cells, Plasmacytoid DC (pDC), T cells, T helper cells, T central memory (Tcm), T effector memory (Tem), T follicular helper (Tfh), T gamma delta (Tgd), Th1 cells, Th17 cells, Th2 cells and Treg

The association between downstream gene expression and enrichment of immune cells was evaluated by Spearman's analysis. Then, the levels of immune cell infiltration were compared for high or low mRNA expression of downstream genes by Wilcoxon rank sum test. $\mathrm{P}<0.05$ is considered as statistically significant.

Results

\section{MiR-211-3p promoted cell proliferation and insensitivity to IC in HSCC}

Firstly, the expression of miR-211-3p in the insensitive tissues of IC were significantly higher than that of sensitive tissues by qRT-PCR (Fig. 1a). Subsequently, we constructed miR-211-3p OE lentivirus to upregulate the expression of miR-211-3p in Fadu cells. The miR-211-3p OE lentivirus increased miR-211$3 p$ expression by 2171.144 times, indicating the high efficiency and stability of the transfection (Fig. 1b). Next, MTT assay showed that the over-expression of miR-211-3p significantly promoted tumor cell growth (Fig. 1c). The IC50 value of paclitaxel in miR-211-3p OE group was significantly higher than NC group, suggesting over-expression of miR-211-3p in Fadu cells induced resistance to IC drugs (Fig. 1d). 
These results illustrated that miR-211-3p played an oncogene role and promoted insensitivity to IC in HSCC.

\section{MiR-211-3p inhibited cell apoptosis and promoted migration in HSCC}

Annexin V-APC-FACS assay demonstrated the number of apoptotic cells was decreased in the miR-211-3p OE group compared to NC group (Fig. 2a). Transwell assay indicated the number of migrated cells was increased in the miR-211-3p OE group compared to NC group (Fig. 2b). These data indicated that overexpression of miR-211-3p could inhibit HSCC cell apoptosis and promote migration.

\section{Prediction and analysis of downstream genes of miR-211- $3 p$}

RNA sequence was performed to screen downstream genes after overexpression of miR-211-3p. A total of 778 genes were identified as DEGs, including 499 up-regulated mRNAs and 279 down-regulated mRNAs (Fig. 3a, b). According to $\mathrm{GO}$ enrichment analysis, the up-regulated genes were mainly enriched in peptidyl-tyrosine phosphorylation, collagen-containing extracellular matrix and receptor ligand activity, whereas the down-regulated genes were enriched in cornification and intermediate filament. According to KEGG pathway analysis, the up-regulated genes were mainly enriched in TNF signaling pathway and MAPK signaling pathway, whereas the down-regulated genes were enriched in estrogen signaling pathway (Fig. 3c, d).

\section{CSF2 and CCL20 were validated as downstream genes of miR-211-3P}

A total of 228 mRNAs were picked out from 499 up-regulated mRNAs to construct the PPI networks (score $>0.7$, high confidence) (Fig. 4a). Eleven candidate genes were chosen by MCODE and further validated in TCGA-HNSCC data (Fig. 4b). CSF2 and CCL20 expression were statistically increased in tumor tissues compared with noncorresponding normal samples and corresponding normal controls (Fig. 4c, d, f, g). In addition, Kaplan-Meier analysis showed significantly better OS rates in patients with lower CSF2 or CCL20 expression than in patients with higher CSF2 or CCL20 expression (Fig. 4e, h, p < 0.05). CSF2 and CCL20 genes involved in TNF signaling pathway were finally identified as downstream genes of miR-211$3 P$.

\section{CSF2 and CCL20 were correlated with immune cell infiltration in HNSCC patients}

The association between the expression level of CSF2 and CCL20 and 24 immune cell types were evaluated by Spearman's analysis (Fig. 5a, b). Scatter diagrams showed that the expression level of CSF2 
were positively correlated with infiltrating levels of Tgd, Th1 cells, neutrophils, DC and Th2 cells, while negatively correlated with cytotoxic cells, B cells, T cells and CD8 T cells $(P<0.05$, Fig. 5 c). CCL20 was positively correlated with CD8 T cells, while negatively correlated with mast cells, iDC, NK cells and DC $(P<0.05$, Fig. $5 d)$. More specifically, we evaluated the infiltration levels of relevant immune cells in distinct CSF2 or CCL20 groups to further validate their correlation (Fig. 5e, f). These data indicated that CSF2 and CCL20 might play oncogenic role by regulating the level of immune cell infiltration in HNSCC.

\section{Discussion}

IC followed by surgery and/or CCR is a comprehensive treatment modality for locally advanced hypopharyngeal cancer patients. However, some patients do not benefit from this treatment due to resistance to IC. So, it is urgently needed to investigate the mechanism of IC resistance. In our study, we provide evidences for the role of miR-211-3p in promoting HSCC progression and identify that miR-211-3p upregulates CSF2/CCL20/TNF signaling to promote IC insensitivity in HSCC.

In HNSCC, previous studies have reported miRNAs take part in its chemoresistance. High level of plasma exosome miR-196a is correlated with poor overall survival and confer cisplatin resistance by downregulating CDKN1B and ING5 in head and neck cancer [16]. MiR-24-3p promotes cell proliferation and regulates chemosensitivity by targeting CHD 5 in HNSCC [17]. In tongue squamous cell carcinoma (TSCC), miR-23a promotes chemoresistance and protects cisplatin-induced apoptosis through inducing Twist expression by a JNK-dependent mechanism [18]. miR-211 is reported to be involved in the development and progression of HNSCC. High expression of miR-211 is associated with poor prognosis in oral carcinoma patients, and significantly promotes tumor cell proliferation, migration, and anchorageindependent colony formation [19]. In oral squamous cell carcinoma (OSCC), miR-211 directly targets TCF12, which leads to FAM213A over-expression and plays oncogenic role [20]. In HNSCC, miR-211 regulates TGF $\beta R I I$ and c-Myc promoting cancer progression [21]. However, studies on the molecular mechanism of miR-211-3p involved in IC resistance are still in a developing stage, a comprehensive exploration is needed urgently.

In our present study, we demonstrated that miR-211-3p was up-regulated in IC insensitive larynxhypopharyngeal tumor tissues, which was consistent with our previous microarray data [22]. We also found that overexpression of miR-211-3p promoted cell proliferation and migration, and inhibited cell apoptosis. The overexpression of miR-211-3p enhanced the IC insensitivity in HSCC cancer cells.

The transcriptome sequencing data of our results showed CSF2 and CCL20 were downstream genes of miR-211-3p. Colony stimulating factor 2 (CSF2), also known as granulocyte macrophage-colony stimulating factor (GM-CSF), acts as a tumor-stimulating factor which promotes immune-independent tumor progression [23]. In gliomas, CSF2 can trigger and drive the alternative activation of tumourinfiltrating microglia/macrophages and shape the tumor immune microenvironment [24]. High CSF2 expression, which is caused by DNA demethylation, affects the immune response to tumor cells and tumor microenvironment. CSF2 plays hub gene role in chemoresistance and correlates worse prognosis 
in colorectal Cancer [25]. CSF2 is a novel pro-inflammatory factor in human gastric cancer, which effectively induces PD-L1 expression on neutrophils via activation of JAK-STAT3 pathway [26]. Besides, C-C motif chemokine ligand 20 (CCL20) promotes chemoresistance and regulate tumor immune microenvironment in many cancers. CCL20 can recruit regulatory T cells to promote chemoresistance via FOX01/CEBPB/NF-KB signaling in colorectal cancer [27]. Chemotherapy-induced CCL20 can activate NF$K B$ pathway and then increase $A B C B 1$ expression, leading to chemoresistance in triple-negative breast cancer patients. The activated NF-KB pathway can also regulate CCL20 expression to further enhance the effect of CCL20 on chemoresistance by a positive feedback loop [28]. Tumor cell-derived CCL20 can promote hepatocellular carcinoma (HCC) progression by interacting with CCR6 highly expressed CD19+CD5+B cells [29]. In lung adenocarcinoma, RUNX3 can upregulate the expression of CCL3 and CCL20 to attract CD8+ T cells into tumor immune microenvironment [30].

In accordance with the literature, we found CSF2 and CCL20 were involved in TNF signaling pathway and correlated with poor prognosis in HNSCC. Meanwhile, the TNF signaling pathway, a typical inflammatory pathway, was activated in HSCC cells after overexpression of miR-211-3p. Furthermore, our results also indicated that CSF2 and CCL2 were correlated with immune cell infiltration. These results suggest that miR-211-3p may promote IC insensitivity via CSF2/CCL20/TNF signaling to regulate tumor immune cell infiltration in HSCC. However, further molecular and cellular experiments are required in the future.

\section{Conclusions}

In summary, as we know, this is the first study to demonstrate that miR-211-3p enhances IC insensitivity and exerts an oncogenic role by upregulating CSF2/CCL20/TNF signaling in HSCC, which may provide new insight in multi-target therapy.

\section{Declarations}

Acknowledgements: We thank all individuals who take part in this research.

\section{Funding}

This project was supported and funded by Beijing Natural Science Foundation Program and Scientific Research Key Program of Beijing Municipal Commission of Education (KZ201910025034); Beijing Municipal Administration of Hospitals' Ascent Plan (DFL20180202); National Key R\&D Program of China (2020YFB1312805); National Natural Science Foundation of China (82002880); Beijing Municipal Administration of Hospitals Incubating Program (PX2021008); and Beijing Hospitals Authority Youth Programme (QML20200205).

\section{Competing Interests}

Financial interests: All authors declare they have no financial interests. The authors declared no potential conflicts of interest with respect to the research, authorship, and/or publication of this article. 


\section{Author Contributions}

All authors contributed to the study conception and design. Material preparation, data collection and analysis were performed by Lingwa Wang, Ru Wang and Tianqiao Huang. The first draft of the manuscript was written by Lingwa Wang, Ru Wang and Jugao Fang, and all authors commented on previous versions of the manuscript. All authors read and approved the final manuscript.

\section{Data Availability}

The datasets generated during and/or analysed during the current study are not publicly available due to the raw data also forms part of an ongoing study, but are available from the corresponding author on reasonable request.

\section{Ethics approval}

No ethical approval is required.

\section{Consent to participate}

Informed consent is not required.

\section{Consent to publish}

Informed consent is not required.

\section{References}

1. Bray F, Ferlay J, Soerjomataram I et al (2018) Global cancer statistics 2018: GLOBOCAN estimates of incidence and mortality worldwide for 36 cancers in 185 countries. CA Cancer J Clin 68(6):394-424. https://doi.org/10.3322/caac.21492

2. Newman JR, Connolly TM, Illing EA et al (2015) Survival trends in hypopharyngeal cancer: a population-based review. Laryngoscope 125(3):624-629. https://doi.org/10.1002/lary.24915

3. Siegel RL, Miller KD, Fuchs HE et al (2021) Cancer Statistics, 2021. CA Cancer J Clin 71(1):7-33. https://doi.org/10.3322/caac.21654

4. Gordin E (2018) Neoadjuvant Chemotherapy for Hypopharyngeal Squamous Cell Carcinoma and Personalized Medicine in Head and Neck Cancer. Ann Surg Oncol 25(4):848-849. https://doi.org/10.1245/s10434-017-6258-8

5. Lu TX, Rothenberg ME (2018) MicroRNA. J Allergy Clin Immunol 141(4):1202-1207. https://doi.org/10.1016/j.jaci.2017.08.034

6. Van Roosbroeck K, Fanini F, Setoyama T et al (2017) Combining Anti-Mir-155 with Chemotherapy for the Treatment of Lung Cancers. Clin Cancer Res 23(11):2891-2904. https://doi.org/10.1158/10780432.CCR-16-1025 
7. Wu K, Hu Y, Yan K et al (2020) microRNA-10b confers cisplatin resistance by activating AKT/mTOR/P70S6K signaling via targeting PPARY in esophageal cancer. J Cell Physiol 235(2):1247-1258. https://doi.org/10.1002/jcp.29040

8. Xue J, Chi Y, Chen Y et al (2016) MiRNA-621 sensitizes breast cancer to chemotherapy by suppressing FBX011 and enhancing p53 activity. Oncogene. 28;35(4):448-58. https://doi.org/10.1038/onc.2015.96

9. Love MI, Huber W, Anders S (2014) Moderated estimation of fold change and dispersion for RNA-seq data with DESeq2. Genome Biol 15(12):550. https://doi.org/10.1186/s13059-014-0550-8

10. Yu G, Wang LG, Han Y et al (2012) clusterProfiler: an R package for comparing biological themes among gene clusters[J]. OMICS 16(5):284-287. https://doi.org/10.1089/omi.2011.0118

11. Wu T, Hu E, Xu S et al (2021) clusterProfiler 4.0: A universal enrichment tool for interpreting omics data[J]. Innov (N Y) 2(3):100141. https://doi.org/10.1016/j.xinn.2021.100141

12. Szklarczyk D, Gable AL, Lyon D et al (2019) STRING v11: protein-protein association networks with increased coverage, supporting functional discovery in genomewide experimental datasets. Nucleic Acids Res 47(D1):D607-D613. https://doi.org/10.1093/nar/gky1131

13. Kohl M, Wiese S, Warscheid B (2011) Cytoscape: software for visualization and analysis of biological networks. Methods Mol Biol 696:291-303. https://doi.org/10.1007/978-1-60761-987-1_18

14. Hänzelmann S, Castelo R, Guinney J (2013) GSVA: gene set variation analysis for microarray and RNA-seq data. BMC bioinformatics 14.1 (2013): 1-15. https://doi.org/10.1186/1471-2105-14-7

15. Bindea G, Mlecnik B, Tosolini M et al (2013) Spatiotemporal dynamics of intratumoral immune cells reveal the immune landscape in human cancer. Immunity 39(4):782-795.

https://doi.org/10.1016/j.immuni.2013.10.003

16. Qin X, Guo H, Wang X et al (2019) Exosomal miR-196a derived from cancer-associated fibroblasts confers cisplatin resistance in head and neck cancer through targeting CDKN1B and ING5. Genome Biol 20(1):12. https://doi.org/10.1186/s13059-018-1604-0

17. Sun X, Xiao D, Xu T et al (2016) miRNA-24-3p promotes cell proliferation and regulates chemosensitivity in head and neck squamous cell carcinoma by targeting CHD5. Future Oncol 12(23):2701-2712. https://doi.org/10.2217/fon-2016-0179

18. Peng F, Zhang H, Du Y et al (2015) miR-23a promotes cisplatin chemoresistance and protects against cisplatin-induced apoptosis in tongue squamous cell carcinoma cells through Twist. Oncol Rep 33(2):942-950. https://doi.org/10.3892/or.2014.3664

19. Chang KW, Liu CJ, Chu TH et al (2008) Association between high miR-211 microRNA expression and the poor prognosis of oral carcinoma. J Dent Res 87(11):1063-1068. https://doi.org/10.1177/154405910808701116

20. Chen YF, Yang CC, Kao SY et al (2016) MicroRNA-211 Enhances the Oncogenicity of CarcinogenInduced Oral Carcinoma by Repressing TCF12 and Increasing Antioxidant Activity. Cancer Res 76(16):4872-4886. https://doi.org/10.1158/0008-5472.CAN-15-1664 
21. Chu TH, Yang CC, Liu CJ et al (2013) miR-211 promotes the progression of head and neck carcinomas by targeting TGFßRII. Cancer Lett 337(1):115-124.

https://doi.org/10.1016/j.canlet.2013.05.032

22. Lian $\mathrm{M}$, Wang $\mathrm{H}$, Fang $\mathrm{J}$ et al (2017) Microarray gene expression analysis of chemosensitivity for docetaxel, cisplatin and 5-fluorouracil (TPF) combined chemotherapeutic regimen in hypopharyngeal squamous cell carcinoma. Chin J Cancer Res 29(3):204-212. https://doi.org/10.21147/j.issn.10009604.2017.03.06

23. Hong IS (2016) Stimulatory versus suppressive effects of GM-CSF on tumor progression in multiple cancer types. Exp Mol Med 48(7):e242. https://doi.org/10.1038/emm.2016.64

24. Sielska M, Przanowski P, Wylot B et al (2013) Distinct roles of CSF family cytokines in macrophage infiltration and activation in glioma progression and injury response. J Pathol 230(3):310-321. https://doi.org/10.1002/path.4192

25. Xu Z, Zhang Y, Xu M et al (2019) Demethylation and Overexpression of CSF2 are Involved in Immune Response, Chemotherapy Resistance, and Poor Prognosis in Colorectal Cancer. Onco Targets Ther 12:11255-11269. https://doi.org/10.2147/OTT.S216829

26. Wang TT, Zhao YL, Peng LS et al (2017) Tumour-activated neutrophils in gastric cancer foster immune suppression and disease progression through GM-CSF-PD-L1 pathway. Gut 66(11):19001911. https://doi.org/10.1136/gutjnl-2016-313075

27. Wang D, Yang L, Yu W et al (2019) Colorectal cancer cell-derived CCL20 recruits regulatory T cells to promote chemoresistance via FOX01/CEBPB/NF-KB signaling. J Immunother Cancer 7(1):215. https://doi.org/10.1186/s40425-019-0701-2

28. Chen W, Qin Y, Wang D et al (2018) CCL20 triggered by chemotherapy hinders the therapeutic efficacy of breast cancer. PLoS Biol 16(7):e2005869. https://doi.org/10.1371/journal.pbio.2005869

29. He H, Wu J, Zang M et al (2017) CCR6+ B lymphocytes responding to tumor cell-derived CCL2O support hepatocellular carcinoma progression via enhancing angiogenesis. Am J Cancer Res 7(5):1151-1163

30. Song Q, Shang J, Zhang C et al (2020) Transcription factor RUNX3 promotes CD ${ }^{+} \mathrm{T}$ cell recruitment by CCL3 and CCL20 in lung adenocarcinoma immune microenvironment. J Cell Biochem 121(56):3208-3220. https://doi.org/10.1002/jcb.29587

\section{Figures}

\section{Figure 1}

MiR-211-3p promoted cell proliferation and insensitivity to IC in HSCC. 

a. qRT-PCR tested the gene expression of miR-211-3p insensitive tumor tissues and insensitive tumor tissues to IC.
b. qRT-PCR analysis of miR-211-3p expression after miR-211-3p OE lentivirus transfection.
c. Cell proliferation was detected through MTT assay.
d. Drug sensitivity testing was determined by MTT assay.

\section{Figure 2}

\section{MiR-211-3p inhibited cell apoptosis and promoted migration in HSCC.}

a. Tumor cell apoptosis was evaluated byAnnexin V-APC-FACS assay.

b. Cell migration was assessed by transwell assay.

\section{Figure 3}

\section{Prediction and analysis of downstream genes of miR-211-3p.}

a. Volcano plot of DEGs. Red: up-regulated DEGs; Blue: down-regulated DEGs.

b. Heatmap of DEGs. Red: up-regulated DEGs; Blue: down-regulated DEGs.

c. Significantly enriched signal terms of up-regulated DEGs in GO and KEGG terms.

d. Significantly enriched signal terms of down-regulated DEGs in GO and KEGG terms.

\section{Figure 4}

\section{CSF2 and CCL20 were identified as downstream genes of miR-211-3P.}

a. The PPI networks of all up-regulated mRNAs were constructed by STRING database.

b. The most significant gene module was chosen by MCODE.

c, f. The expression of CSF2 and CCL20 in tumor tissues and non-corresponding normal samples.

d, g. The expression of CSF2 and CCL20 in tumor tissues and corresponding normal samples. 
e, h. Kaplan-Meier survival analysis of HNSCC patients using the log-rank test. Kaplan-Meier analysis demonstrated that CSF2 and CCL20 overexpression were prognostic factor for LSCC patients.

\section{Figure 5}

\section{CSF2 and CCL20 were correlated with immune cell infiltration in HNSCC patients}

a. Relationships among infiltration levels of 24 immune cell types and CSF2 expression.

b. Relationships among infiltration levels of 24 immune cell types and CCL20 expression.

c. Scatter diagrams of the correlation of CSF2 expression level with enrichment of immune cells.

d. Scatter diagrams of the correlation of CCL20 expression level with enrichment of immune cells.

e. The comparison of infiltration levels of most correlated immune cells under high or low CSF2 expression subgroup.

f. The comparison of infiltration levels of most correlated immune cells under high or low CCL20 expression subgroup. 